\title{
Palladium-Catalyzed Cross-Coupling of Aryl Triethylammonium Bis(catechol) Silicates with Aryl Bromides Using Microwave Irradiation
}

\author{
Supporting Information
}

W. Michael Seganish and Philip DeShong

\section{Table of Contents}

$\begin{array}{ll}\text { General Experimental } & \text { S2 }\end{array}$

General Procedure for the Palladium-Catalyzed Coupling Reaction S2

$\begin{array}{ll}\text { Characterization information for all coupled products } & \text { S2-S5 }\end{array}$

References $\quad$ S5

${ }^{1} \mathrm{H}$ NMR spectrum for biphenyl $\quad$ S6

$\begin{array}{ll}{ }^{1} \mathrm{H} \text { NMR spectrum for 2-methoxybiphenyl } & \text { S7 }\end{array}$

${ }^{1} \mathrm{H}$ NMR spectrum for 3-methoxybiphenyl $\quad$ S8

${ }^{1} \mathrm{H}$ NMR spectrum for 4-methoxybiphenyl $\quad$ S9

$\begin{array}{ll}{ }^{1} \mathrm{H} \text { NMR spectrum for 2-methylbiphenyl } & \text { S10 }\end{array}$

$\begin{array}{ll}{ }^{1} \mathrm{H} \text { NMR spectrum for 2,6-dimethylbiphenyl } & \text { S11 }\end{array}$

$\begin{array}{ll}{ }^{1} \mathrm{H} \text { NMR spectrum for 4-tert-butylbiphenyl } & \mathrm{S} 12\end{array}$

$\begin{array}{ll}{ }^{1} \mathrm{H} \text { NMR spectrum for 4-acetylbiphenyl } & \mathrm{S} 13\end{array}$

$\begin{array}{ll}{ }^{1} \mathrm{H} \text { NMR spectrum for 4-nitrobiphenyl } & \text { S14 }\end{array}$

$\begin{array}{ll}{ }^{1} \mathrm{H} \text { NMR spectrum for 1-phenyl napthalene } & \text { S15 }\end{array}$

${ }^{1} \mathrm{H}$ NMR spectrum for 2־methoxy-biphenyl-4-carboxylic acid ethyl ester $\quad$ S16

$\begin{array}{ll}{ }^{1} \mathrm{H} \text { NMR spectrum for 2,3,4-trimethoxybiphenyl } & \mathrm{S} 17\end{array}$

${ }^{1} \mathrm{H}$ NMR spectrum for 2,3,4-trimethoxy-6-carbaldehyde-biphenyl S18

${ }^{1} \mathrm{H}$ NMR spectrum for 2,3,4-trimethoxy-6-carbomethoxy-biphenyl S19

${ }^{1} \mathrm{H}$ NMR spectrum for 2-methoxy-5-phenylcyclohepta-2,4,6-trien-1-one $\quad$ S20

${ }^{1} \mathrm{H}$ NMR spectrum for 1-bromo-2,3,4,-trimethoxybenzene $\quad$ S21 


\section{General Experimental}

Thin-layer chromatography (TLC) was performed on $0.25 \mathrm{~mm}$ Merck silica gel coated plates treated with a UV-active binder with compounds being identified by one or more of the following methods: UV (254 nm), iodine, or vanillin/sulfuric acid charring. Flash chromatography was performed using thick walled columns and medium pressure silica gel (Whatman 200-425 mesh), with column length and diameter being determined by the method of Still. ${ }^{1}$ Melting points were taken in Kimax soft glass capillary tubes using a Thomas-Hoover Uni-Melt capillary melting point apparatus (Model 6406K) equipped with a calibrated thermometer. Melting points are corrected. Infrared spectra band positions are reported in reciprocal centimeters $\left(\mathrm{cm}^{-1}\right)$ and relative intensities are listed as br (broad), s (strong), $\mathrm{m}$ (medium), or w (weak). Nuclear magnetic resonance $\left({ }^{1} \mathrm{H},{ }^{13} \mathrm{C}\right.$ NMR) spectra were recorded on a $400 \mathrm{MHz}$ spectrometer. Chemical shift values are reported in parts per million (\) relative to tetramethylsilane (TMS). Coupling constants ( $J$ values) are reported in hertz $(\mathrm{Hz})$, and spin multiplicities are indicated by the following symbols: $\mathrm{s}$ (singlet), d (doublet), $\mathrm{t}$ (triplet), q (quartet), $\mathrm{m}$ (multiplet). Low resolution mass spectrometry (LRMS) and high resolution mass spectrometry (HRMS) data are reported in the form of $\mathrm{m} / \mathrm{z}$ (intensity relative to base peak $=100)$. The matrix used for fast atom bombardment $(\mathrm{FAB})$ was ethylene glycol. Tetrahydrofuran was distilled from sodium/benzophenone ketyl prior to use.

Bis(dibenzylideneacetone)palladium ( $\left.\mathrm{Pd}(\mathrm{dba})_{2}\right)$, and tetrabutylammonium fluoride (1M solution in THF) were purchased from Acros and used as received. 2-(Dicyclohexylphosphino)biphenyl was purchased from Strem and recrystllized from ethanol prior to use.

Microwave reactions were carried out on a CEM Discover microwave reactor with a circular, single mode, self tuning microwave applicator operating at $2450 \mathrm{MHz}$. The reaction vessel was purged with argon prior to the addition of reagents.

All compounds were determined to be $>95 \%$ pure by ${ }^{1} \mathrm{H}$ NMR or GC analysis, unless otherwise noted. Previously reported compounds were characterized via ${ }^{1} \mathrm{H}$ and ${ }^{13} \mathrm{C}$ NMR and IR and compared to literature values.

\section{General procedure for the palladium catalyzed cross-coupling reaction of aryl bromides with aryl bis(catechol) silicates:}

Aryl bromide (1.00 mmol), aryl bis (catechol) silicate $(1.50 \mathrm{mmol}), \mathrm{Pd}(\mathrm{dba})_{2}(0.05 \mathrm{mmol})$ and dicyclohexylphosphinobiphenyl $(0.05 \mathrm{mmol})$ were combined in a microwave tube with a magnetic stir bar. The microwave tube was sealed with a septum and $5 \mathrm{~mL}$ of THF was added, followed by TBAF (1.0 M in THF, $1.50 \mathrm{mmol}$ ). The reaction was stirred briefly to dissolve the reagents and placed into the microwave reactor. The reaction was then heated to a set temperature of $120^{\circ} \mathrm{C}(50 \mathrm{~W})$ for $10 \mathrm{~min}$. The tube was removed from the reactor and the contents filtered through a short plug $(2 \mathrm{~cm})$ of silica gel $\left(\mathrm{CH}_{2} \mathrm{Cl}_{2}\right.$ elutant). The solvent was removed in vacuo and the biaryl product purified via column chromatography. 
Biphenyl. Column chromatography ( $T L C \mathrm{R}_{\mathrm{f}}=0.54$, pentane) yielded a white solid (89\%) $\mathrm{mp} 69-71{ }^{\circ} \mathrm{C}$ (lit. $\left.69-70^{\circ} \mathrm{C}\right)$. The ${ }^{1} \mathrm{H}$ and ${ }^{13} \mathrm{C}$ NMR and IR match that reported by Koza. ${ }^{2}$

2-Methoxybiphenyl. Column chromatography (19:1 hexanes/EtOAc, TLC $\left.R_{f}=0.37\right)$ yielded a colorless oil (84\%). The spectral data (IR, ${ }^{1} \mathrm{H}$ and ${ }^{13} \mathrm{C}$ NMR) were identical to those previously reported by Buchwald. $^{3}$

3-Methoxybiphenyl (Table 7, Entry 5). Column chromatography (19:1 hexanes/EtOAc, TLC $\mathrm{R}_{\mathrm{f}}=0.21$ ) yielded a colorless oil (84\%); IR (CCl 3064 (m), 3033 (m), 3005 (w), 2957 (m), 2940 (m), 2833 (m), 1604, (s), 1569 (s), 1480 (s), 1424 (m), 1293 (m), 1214 (s), 1176 (m), 1058 (m); ${ }^{13} \mathrm{C}$ NMR $\left(\mathrm{CDCl}_{3}\right) \square 55.1$, 112.6, 112.6, 112.9, 119.6, 127.2, 127.4, 128.7, 129.7, 141.1, 142.7, 159.9; LRMS (EI $\left.{ }^{+}\right) \mathrm{m} / \mathrm{z} 184\left(\mathrm{M}^{+}, 100\right)$, 154 (22), 141 (46), 139 (18), 115 (42); HRMS ( $\left.\mathrm{EI}^{+}\right) \mathrm{m} / \mathrm{z}$ calcd for $\mathrm{C}_{13} \mathrm{H}_{12} \mathrm{O}$ 184.0888, found 184.0884. The spectral data $\left({ }^{1} \mathrm{H}\right.$ NMR) was identical to that previously reported by Nielson. ${ }^{4}$

4-Methoxybiphenyl. Column chromatography (19:1 hexanes/EtOAc, TLC $\left.\mathrm{R}_{\mathrm{f}}=0.32\right)$ yielded a white solid (81\%) mp 87.0-87.5 ${ }^{\circ} \mathrm{C}$ (lit. 87.5 ${ }^{\circ} \mathrm{C}$ ). ${ }^{5}$ The spectral data (IR, ${ }^{1} \mathrm{H}$ and ${ }^{13} \mathrm{C}$ NMR) were identical to those previously reported by DeShong. ${ }^{5}$

2-Methylbiphenyl. Column chromatography (pentane, $T L C \mathrm{R}_{\mathrm{f}}=0.57$ ) yielded a colorless oil (86\%). The spectral data (IR, ${ }^{1} \mathrm{H}$ and ${ }^{13} \mathrm{C} \mathrm{NMR}$ ) were identical to those previously reported by DeShong. ${ }^{5}$

2,6-Dimethylbiphenyl. Column chromatography (pentane, TLC $\mathrm{R}_{\mathrm{f}}=0.47$ ) yielded a colorless oil $(80 \%)$; IR ( $\left.\mathrm{CCl}_{4}\right)$ 3029, (w), 2992 (w), 2950 (w), 2916 (w), 2829 (w), 1611 (m), 1500 (m), 1241 (m), 1176 (w), 1045 (w). The spectral data $\left({ }^{1} \mathrm{H}\right.$ and ${ }^{13} \mathrm{C}$ NMR) were identical to those previously reported by Buchwald. ${ }^{3}$

4-tert-Butylbiphenyl (Table 4, Entry 8). Column chromatography (pentane, TLC $\mathrm{R}_{\mathrm{f}}=0.36$ ) yielded a white solid (88\%) mp 49-50 ${ }^{\circ} \mathrm{C}$ (lit. $47-49^{\circ} \mathrm{C}$ ). ${ }^{3}$ The spectral data (IR, ${ }^{1} \mathrm{H}$ and ${ }^{13} \mathrm{C}$ NMR) were identical to those previously reported by Buchwald. ${ }^{3}$

4-Acetylbiphenyl. Column chromatography (9:1 hexanes/EtOAc, TLC $\left.\mathrm{R}_{\mathrm{f}}=0.31\right)$ yielded a white solid (89\%) mp 119.0-119.4 ${ }^{\circ} \mathrm{C}$ (lit. 119.0-119.5 ${ }^{\circ} \mathrm{C}$ ). ${ }^{5}$ The spectral data (IR, ${ }^{1} \mathrm{H}$ and ${ }^{13} \mathrm{C}$ NMR) were identical to those previously reported by DeShong. ${ }^{5}$

4-Nitrobiphenyl. Column chromatography (4:1 hexanes/EtOAc, TLC $\left.\mathrm{R}_{\mathrm{f}}=0.20\right)$ yielded a white solid (93\%) mp 114-115 ${ }^{\circ} \mathrm{C}$ (lit. 113-115 $\left.{ }^{\circ} \mathrm{C}\right)$; $^{6} \mathrm{IR}\left(\mathrm{CCl}_{4}\right) 3064$ (w), 3036 (w), 1600 (m), 1552 (m), 1521 (s), 1476 (w), 1341 (s). The spectral data $\left({ }^{1} \mathrm{H}\right.$ and ${ }^{13} \mathrm{C}$ NMR) were identical to those previously reported by Novak. $^{6}$ 
1-Phenylnapthalene. Column chromatography (pentane, $T L C \mathrm{R}_{\mathrm{f}}=0.42$ ) yielded a colorless oil (84\%); LRMS (EI $\left.{ }^{+}\right) \mathrm{m} / \mathrm{z} 204\left(\mathrm{M}^{+}, 100\right), 203$ (74), 188 (20), 29 (50), 18 (90); HRMS (EI $\left.{ }^{+}\right) \mathrm{m} / \mathrm{z}$ calcd for $\mathrm{C}_{16} \mathrm{H}_{12}$ 204.0939, found 204.0948. The IR and ${ }^{1} \mathrm{H}$ NMR were identical to those reported by DeShong. ${ }^{5}$ The ${ }^{13} \mathrm{C}$ NMR was identical to that reported by Bergbreiter. ${ }^{7}$

2EMethoxy-biphenyl-4-carboxylic acid ethyl ester. Column chromatography (4:1 hexanes/EtOAc, TLC $\mathrm{R}_{\mathrm{f}}=0.53$ ) yielded a white solid (82\%) mp 104.5-105.0 ${ }^{\circ} \mathrm{C}\left(\mathrm{lit} .105{ }^{\circ} \mathrm{C}\right) ;^{8} \mathrm{IR}\left(\mathrm{CCl}_{4}\right) 3040(\mathrm{w}), 2985(\mathrm{w})$, 2957 (w), 2933 (w), 2874 (w), 2833 (w), 1718 (s), 1604 (m), 1500 (m), 1462 (w), 1276 (s), 1245 (m), 1183 (m), $1107(\mathrm{~m}), 1045(\mathrm{~m})$. The spectral data $\left({ }^{1} \mathrm{H}\right.$ and ${ }^{13} \mathrm{C}$ NMR) were identical to those previously reported by Gosmimi. ${ }^{9}$

2,3,4-Trimethoxybiphenyl. Column chromatography (TLC $\mathrm{R}_{\mathrm{f}}=0.31,9: 1$ Hexanes/EtOAc) yielded a yellow oil which was recrystallized (hexane) to give a white solid (84\%) mp 47.2-47.9 ${ }^{\circ} \mathrm{C}$ (lit. $46-47{ }^{\circ} \mathrm{C}$ ). The ${ }^{1} \mathrm{H}$, and ${ }^{13} \mathrm{C}$ NMR, and IR match that reported by Banwell. ${ }^{10}$

2,3,4-Trimethoxy-6-carbaldehyde-biphenyl. Column chromatography $\left(\mathrm{TLC}_{\mathrm{f}}=0.24,9: 1\right.$ Hexanes/EtOAc) yielded a white solid (74\%) mp 91.2-92.4 ${ }^{\circ} \mathrm{C}$. IR ( $\left.\mathrm{CCl}_{4}\right) 3085$ (w) 3067 (w), 3009 (w), 2960 (m), 2933 (m), 2857 (m), 1683 (s), 1587 (m), 1328 (s), $1193(\mathrm{~m}), 1141$ (s), 1096 (s); ${ }^{1} \mathrm{H} \mathrm{NMR}\left(\mathrm{CDCl}_{3}\right)$ $\mathrm{\square} 3.59$ (s, 3H), 3.94 (s, 3H), 3.99 (s, 3H), 7.30-7.35 (m, 3H), 7.42-7.43 (m, 2H), 9.63 (s, $1 \mathrm{H}) ;{ }^{13} \mathrm{C} \mathrm{NMR}$ $\left(\mathrm{CDCl}_{3}\right) \square 56.6,61.4,61.5,105.6,110.2,127.5,128.1,123.3,128.4,129.9,130.1,131.4,133.2,191.8$; LRMS (FAB ${ }^{+}$) m/z $273\left(\mathrm{M}^{+}+\mathrm{H}, 100\right), 272$ (80), 255 (42), 230 (24), 214 (19), 151 (18), 135 (24), 119 (25), 85 (29), 55 (34), 43 (25); HRMS (FAB ${ }^{+}, \mathrm{M}^{+}$) m/z calcd for $\mathrm{C}_{16} \mathrm{H}_{16} \mathrm{O}_{4} 272.1049$, found 272.1045 .

2,3,4-Trimethoxy-6-carbomethoxy-biphenyl. Column chromatography $\left(\mathrm{TLC}_{\mathrm{f}}=0.31,4: 1\right.$ Hexanes/EtOAc) yielded a white solid (79\%) mp 67.2-67.8 ${ }^{\circ} \mathrm{C}$. IR $\left(\mathrm{CCl}_{4}\right) 3065$ (w), $3002(\mathrm{w}), 2940$ (m), 2836 (w), 1718 (s), 1593 (m), 1486 (m), 1435 (m), 1397 (m), 1335 (s), 1224 (m), 1134 (m), 1100 (s), 1045 (m), 1007 (m); ${ }^{1} \mathrm{H} \mathrm{NMR}\left(\mathrm{CDCl}_{3}\right) \square 3.54$ (s, 3H), 3.57 (s, 3H), 3.94 (s, 3H), $3.96(\mathrm{~s}, 3 \mathrm{H}), 7.22-7.26(\mathrm{~m}, 3 \mathrm{H})$, 7.37-7.38 (m, 3H); ${ }^{13} \mathrm{C}$ NMR $\left(\mathrm{CDCl}_{3}\right) \square 51.9,56.1,59.9,60.0,108.9,126.4,126.9,127.6,129.3,130.7$, 136.7, 145.3, 151.5, 152.3, 168.1; LRMS (FAB ${ }^{+}$) m/z $302\left(\mathrm{M}^{+}, 100\right), 271$ (79), 256 (20); HRMS (FAB ${ }^{+}$, $\mathrm{M}^{+}$) $\mathrm{m} / \mathrm{z}$ calcd for $\mathrm{C}_{17} \mathrm{H}_{20} \mathrm{O}_{5} 302.1154$, found 302.1148 .

2-Methoxy-5-phenylcyclohepta-2,4,6-trien-1-one. 5-Bromotropolone was prepared according to a literature procedure. ${ }^{11}$ Column chromatography $\left(\mathrm{TLC} \mathrm{R}_{\mathrm{f}}=0.38\right.$, EtOAc) yielded a light tan solid (76\%) mp 139.2-140.4 ${ }^{\circ} \mathrm{C}$ (lit. 140-141 $\left.{ }^{\circ} \mathrm{C}\right) .{ }^{12}$ The IR, ${ }^{1} \mathrm{H}$ NMR, and ${ }^{13} \mathrm{C}$ NMR matched that reported by Banwell. $^{12}$ 
1-Bromo-2,3,4,-trimethoxybenzene. Pyridinium tribromide (90\%) (4.66 g, $13.1 \mathrm{mmol})$, was added in small portions over $30 \mathrm{~min}$ to a light red solution of 1,2,3-trimethoxybenzene (2.00 g, $11.9 \mathrm{mmol})$ and $\mathrm{FeCl}_{3} \bullet 6 \mathrm{H}_{2} \mathrm{O}(50 \mathrm{mg} 0.18 \mathrm{mmol})$ in $\mathrm{Ac}_{2} \mathrm{O}(35 \mathrm{~mL})$. The deep red solution was then heated at $55^{\circ} \mathrm{C}$ for 5 min. The reaction was carefully poured into $100 \mathrm{~mL}$ of hot water. After cooling, the mixture was extracted with $50 \mathrm{~mL}$ of $\mathrm{Et}_{2} \mathrm{O}(\mathrm{x} 4)$, dried $\left(\mathrm{MgSO}_{4}\right)$, and evaporated to yield a yellow oil. Column chromatography $\left(\mathrm{TLC} \mathrm{R}_{\mathrm{f}}=0.44,4: 1\right.$ hexanes/EtOAc) produced a pale yellow oil (82\%). IR $\left(\mathrm{CCl}_{4}\right) 3009(\mathrm{~m}), 2960(\mathrm{~m})$, 2940 (s), 2898 (m), 2836 (m), 1580 (m), 1486 (s), 1462 (s), 1414 (s), 1293 (s), 1221 (s), 1096 (s), 1017 (s).

${ }^{13} \mathrm{C} \mathrm{NMR}\left(\mathrm{CDCl}_{3}\right)$ д 56.2, 61.0, 61.1, 108.4, 108.6, 126.8, 151.0, 153.3. The ${ }^{1} \mathrm{H}$ NMR matches that reported by Pettit. ${ }^{13}$

\section{References}

(1) Still, W. C.; Kahn, M.; Mitra, A. J. Org. Chem. 1978, 43, 2923-2925.

(2) Koza, D. J.; Carita, E. Synthesis 2002, 2183-2186.

(3) Wolfe, J. P.; Singer, R. A.; Yang, B. H.; Buchwald, S. L. J. Am. Chem. Soc. 1999, 121, 9550-9561.

(4) Nielsen, S. F.; Peters, D.; Axelsson, O. Synth. Commun. 2000, 30, 3501-3509.

(5) Mowery, M. E.; DeShong, P. J. Org. Chem. 1999, 64, 3266-3270.

(6) Wallow, T. I.; Novak, B. M. J. Org. Chem. 1994, 59, 5034-5037.

(7) Bergbreiter, D. E.; Osburn, P. L.; Wilson, A.; Sink, E. M. J. Am. Chem. Soc. 2000, 122, 9058-9064.

(8) Darses, S.; Jeffery, T.; Brayer, J. L.; Demoute, J. P.; Genet, J. P. Bull. Soc. Chim. Fr. 1996, 133, 1095-1102.

(9) Gomes, P.; Fillon, H.; Gosmini, C.; Labbe, E.; Perichon, J. Tetrahedron 2002, 58, 84178424.

(10) Banwell, M. G.; Herbert, K. A.; Buckleton, J. R.; Clark, G. R.; Rickard, C. E. F.; Lin, C. M.; Hamel, E. J. Org. Chem. 1988, 53, 4945-4952.

(11) Banwell, M. G.; Lambert, J. N.; Reum, M. E.; Onrust, R. Org. Prep. Proced. Int. 1988, 20, 393-399.

(12) Banwell, M. G.; Cameron, J. M.; Collis, M. P.; Gravatt, G. L. Aust. J. Chem. 1997, 50, 395-407.

(13) Pettit, G. R.; Grealish, M. P.; Herald, D. L.; Boyd, M. R.; Hamel, E.; Pettit, R. K. J. Med. Chem. 2000, 43, 2731-2737. 

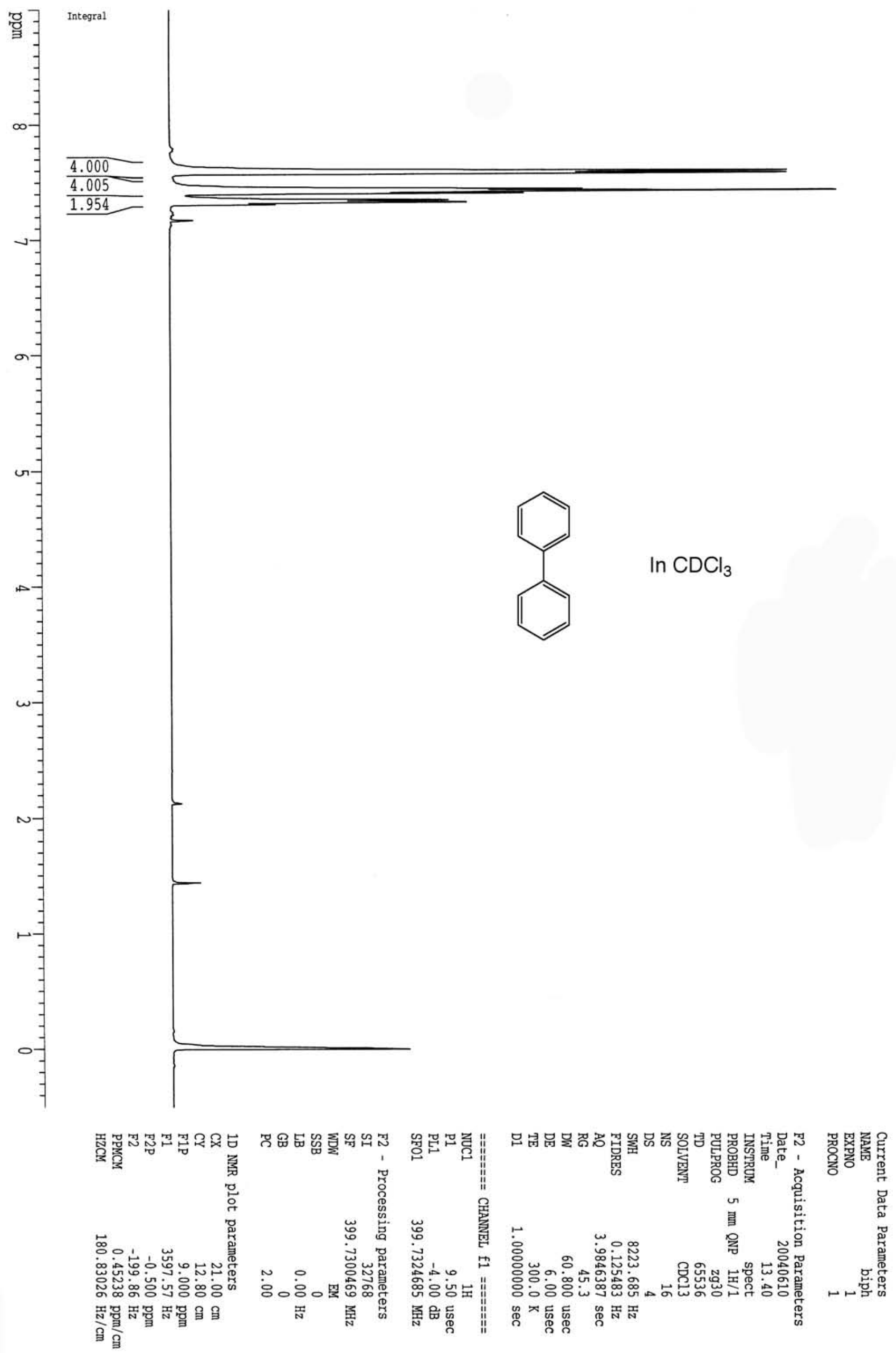


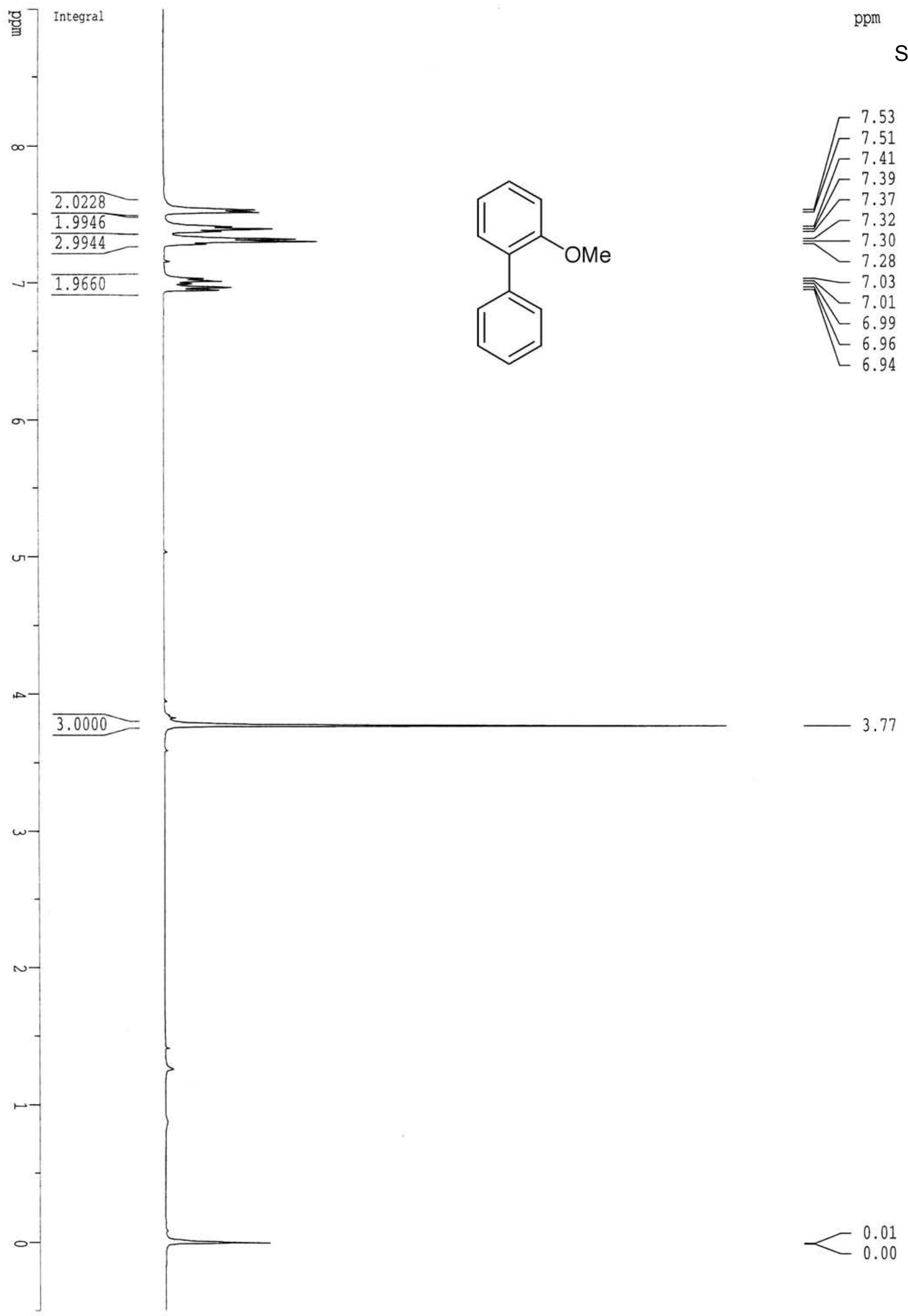




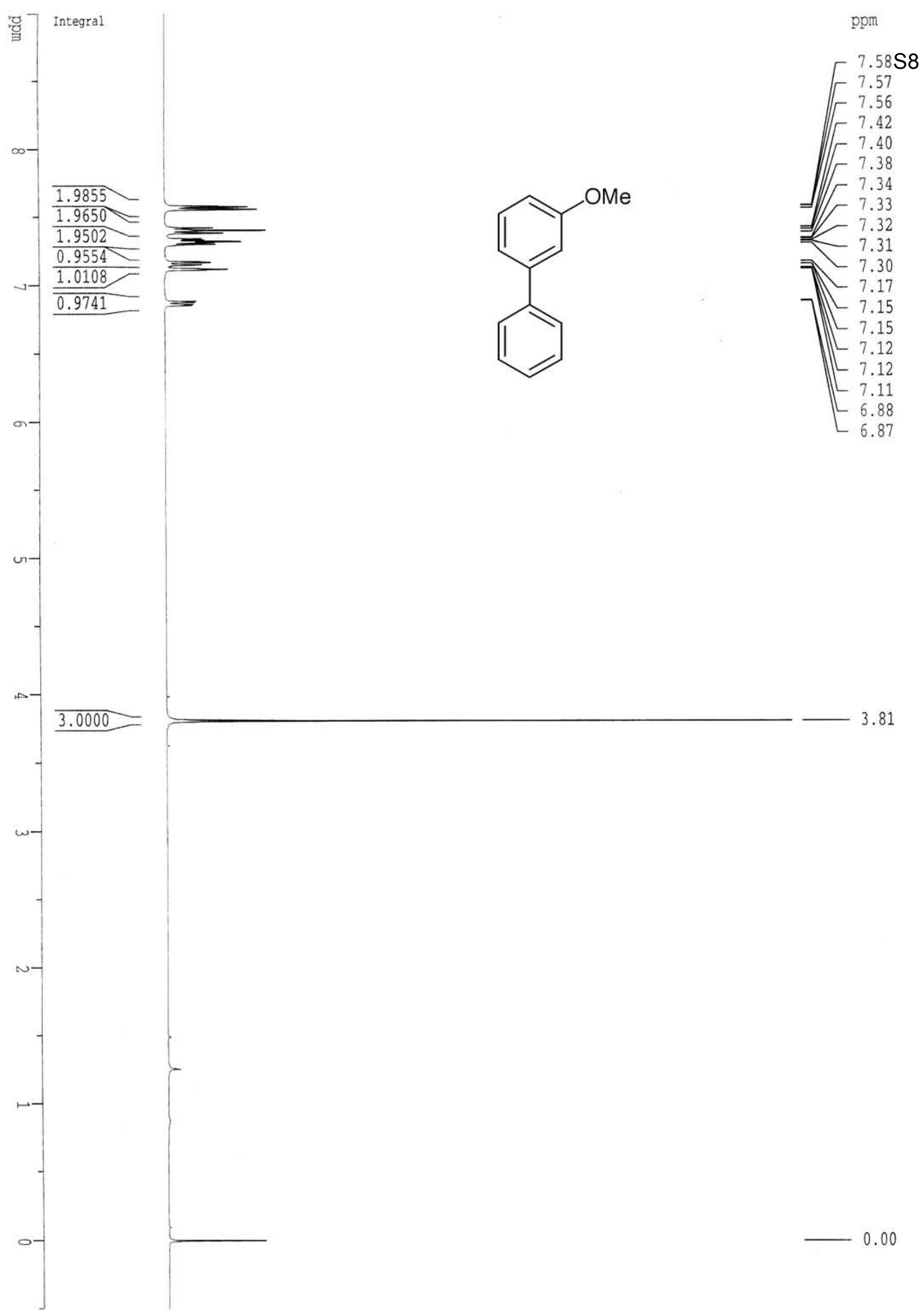




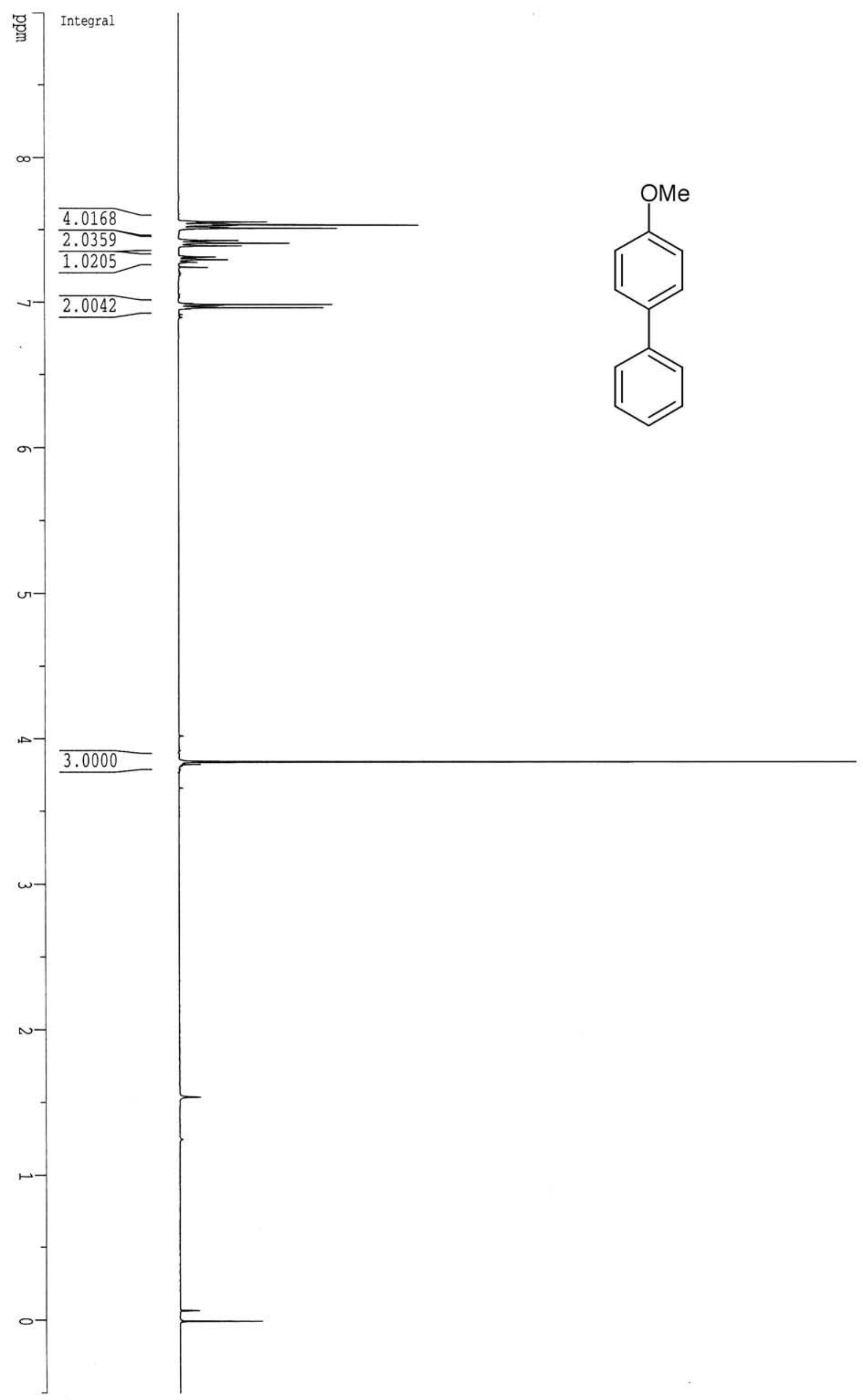



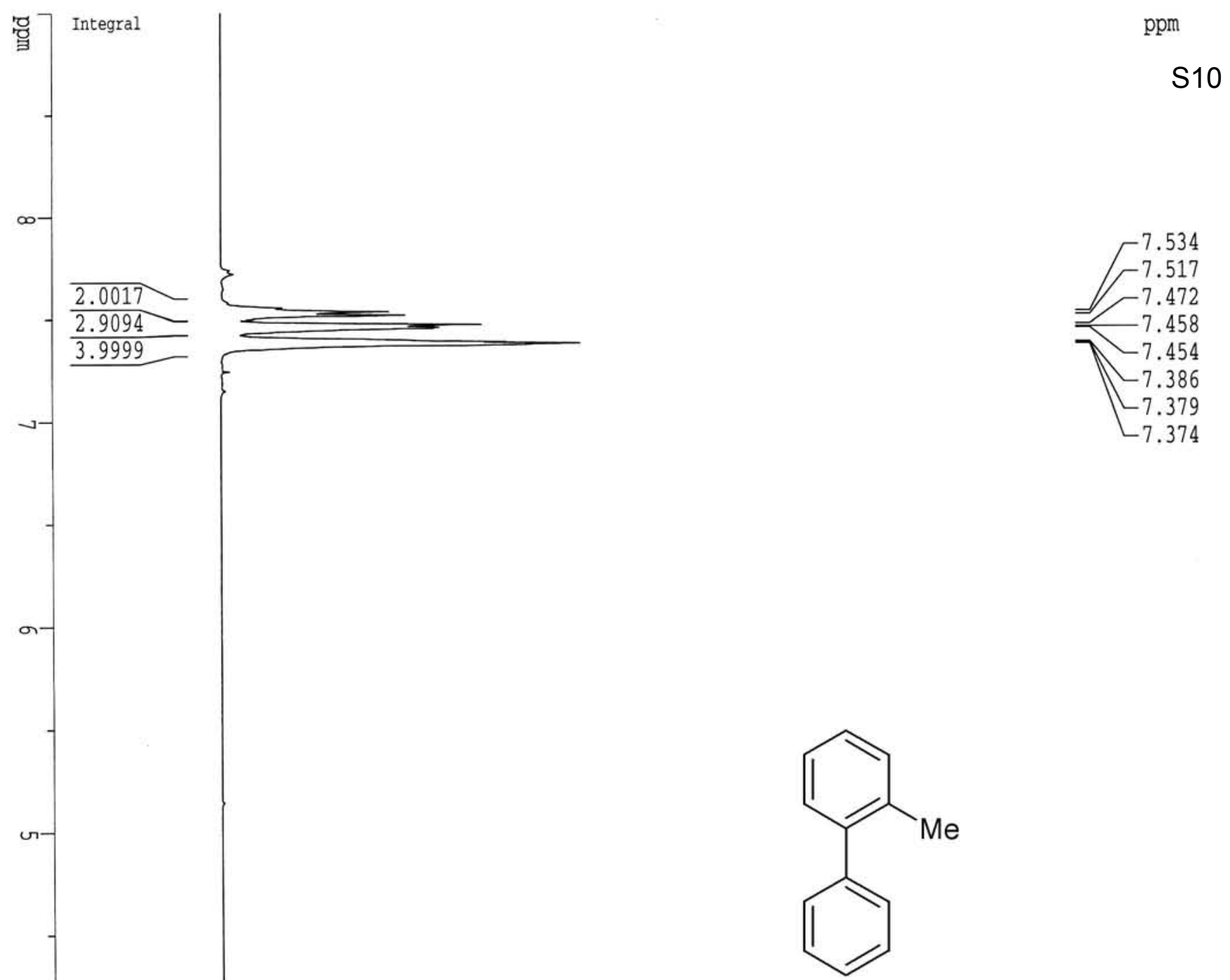

\subsection{0}

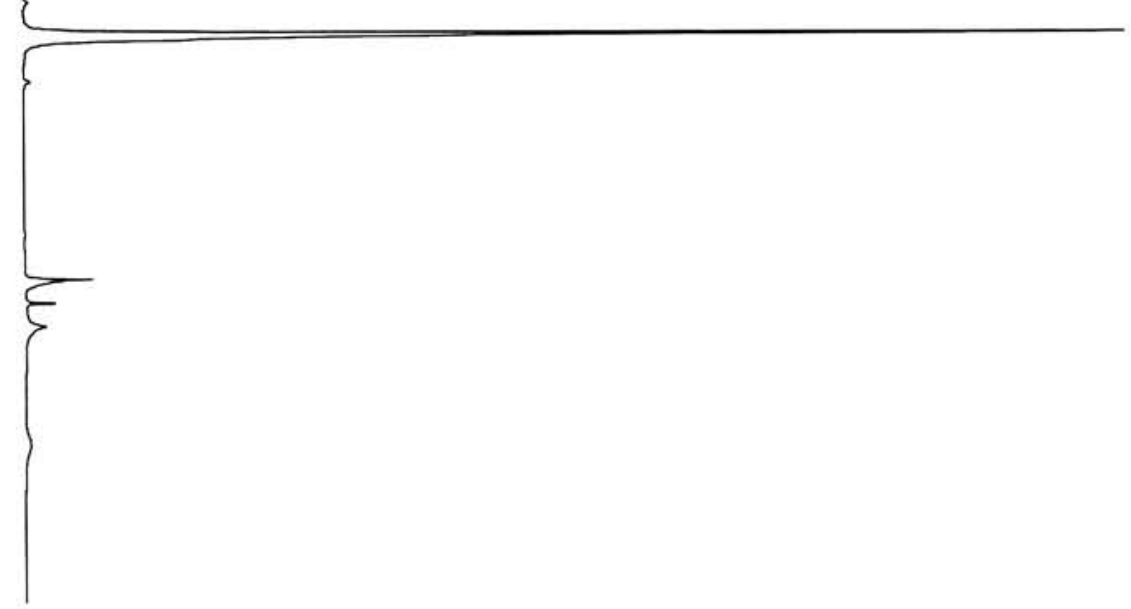




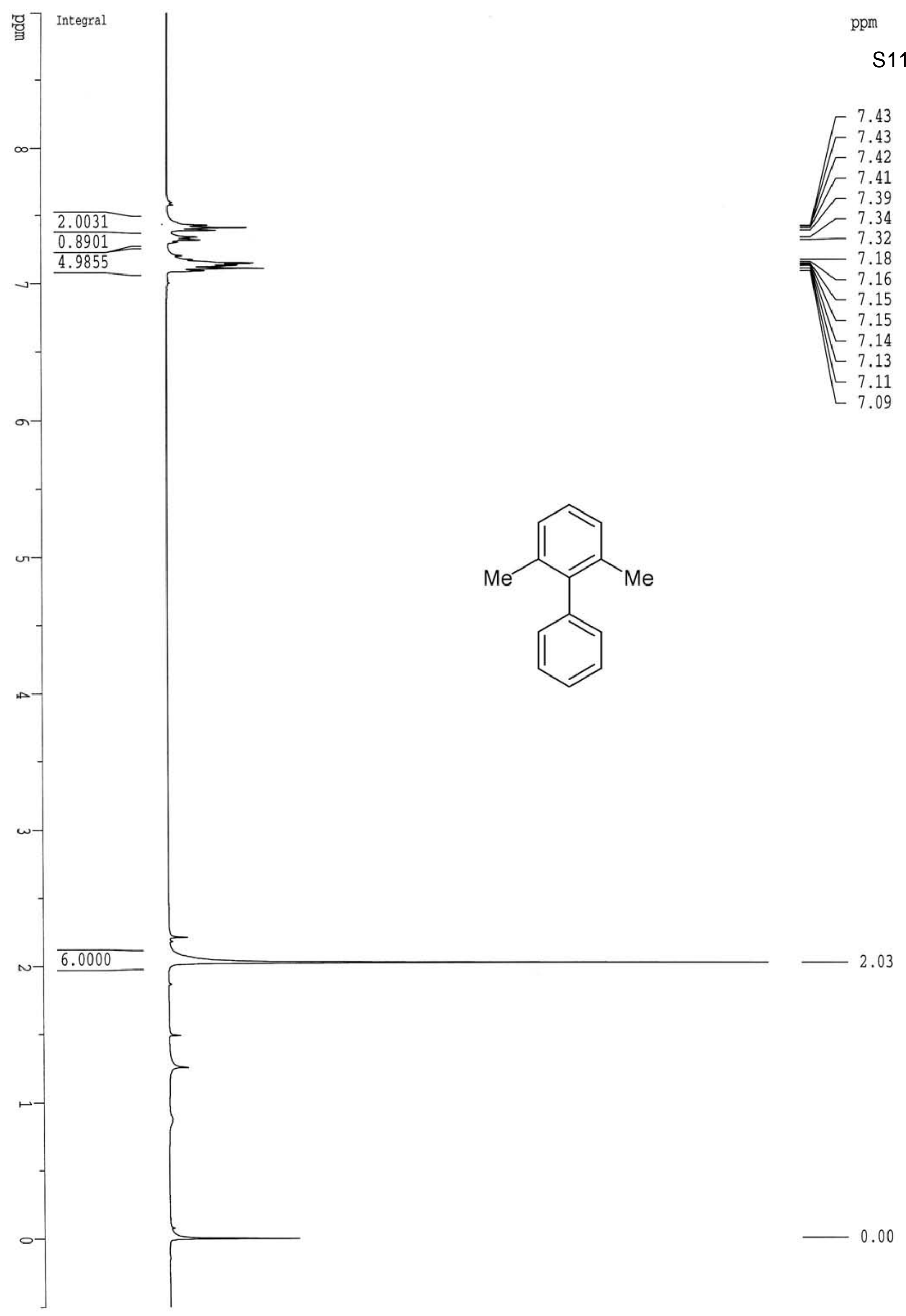



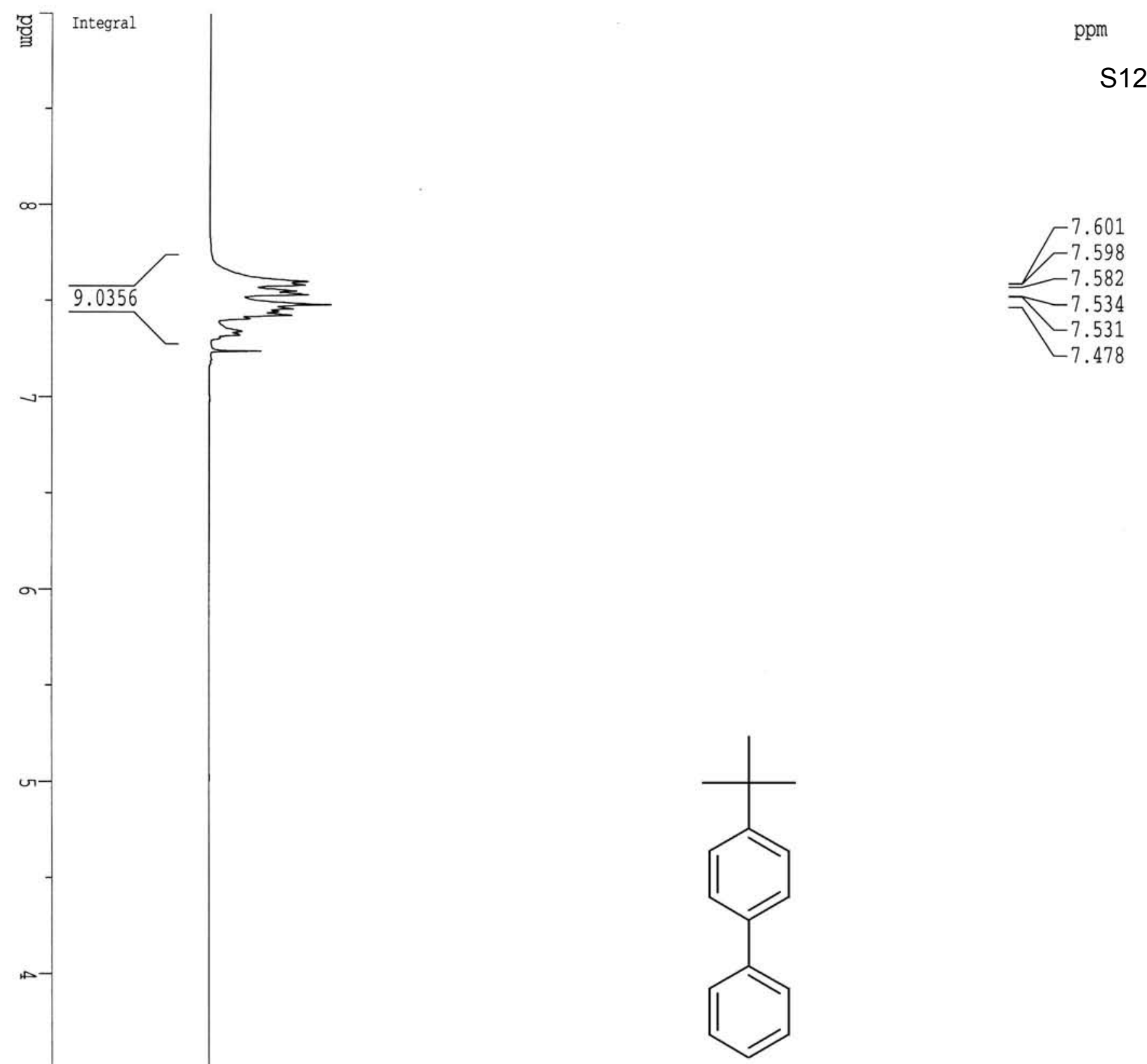


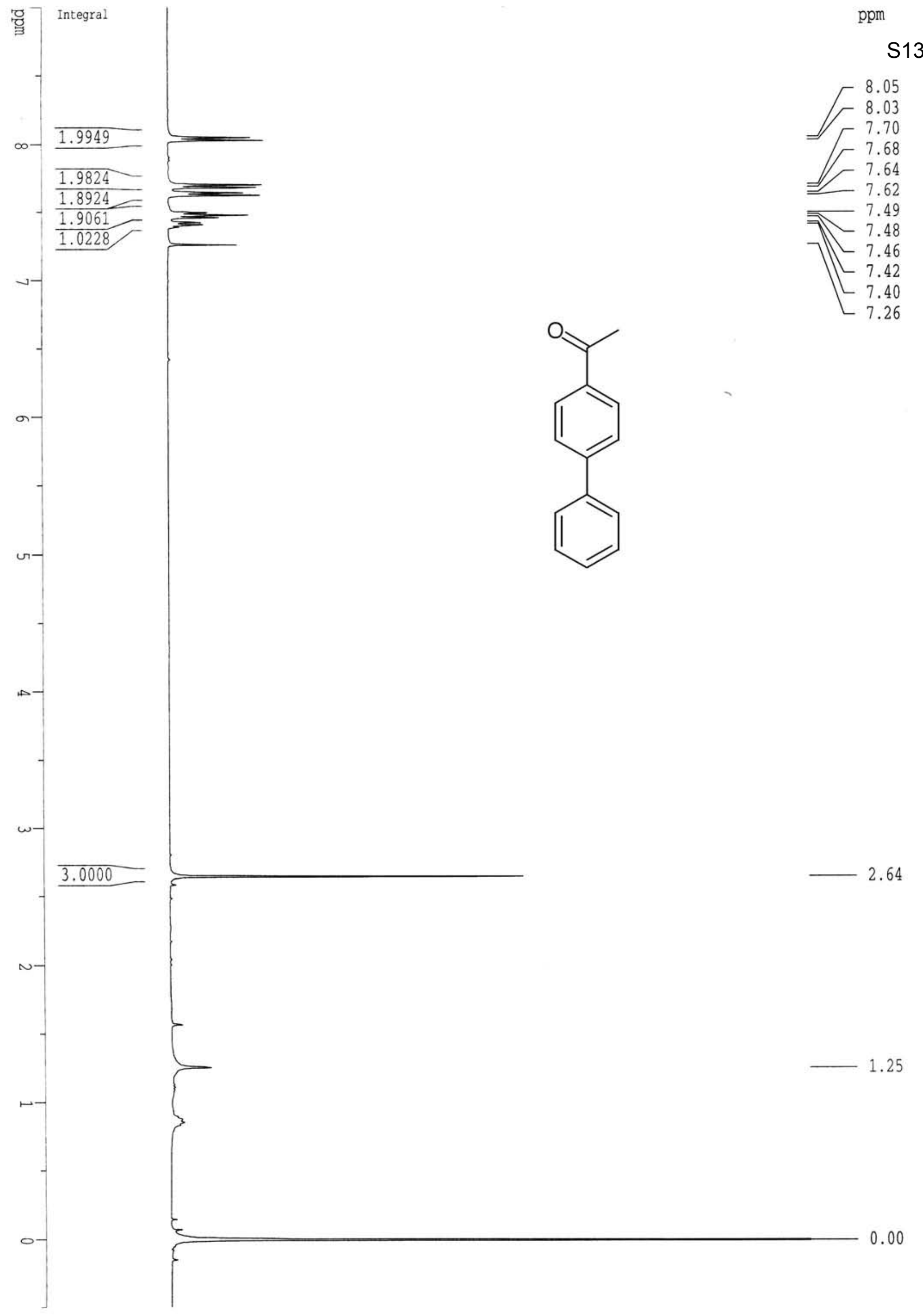




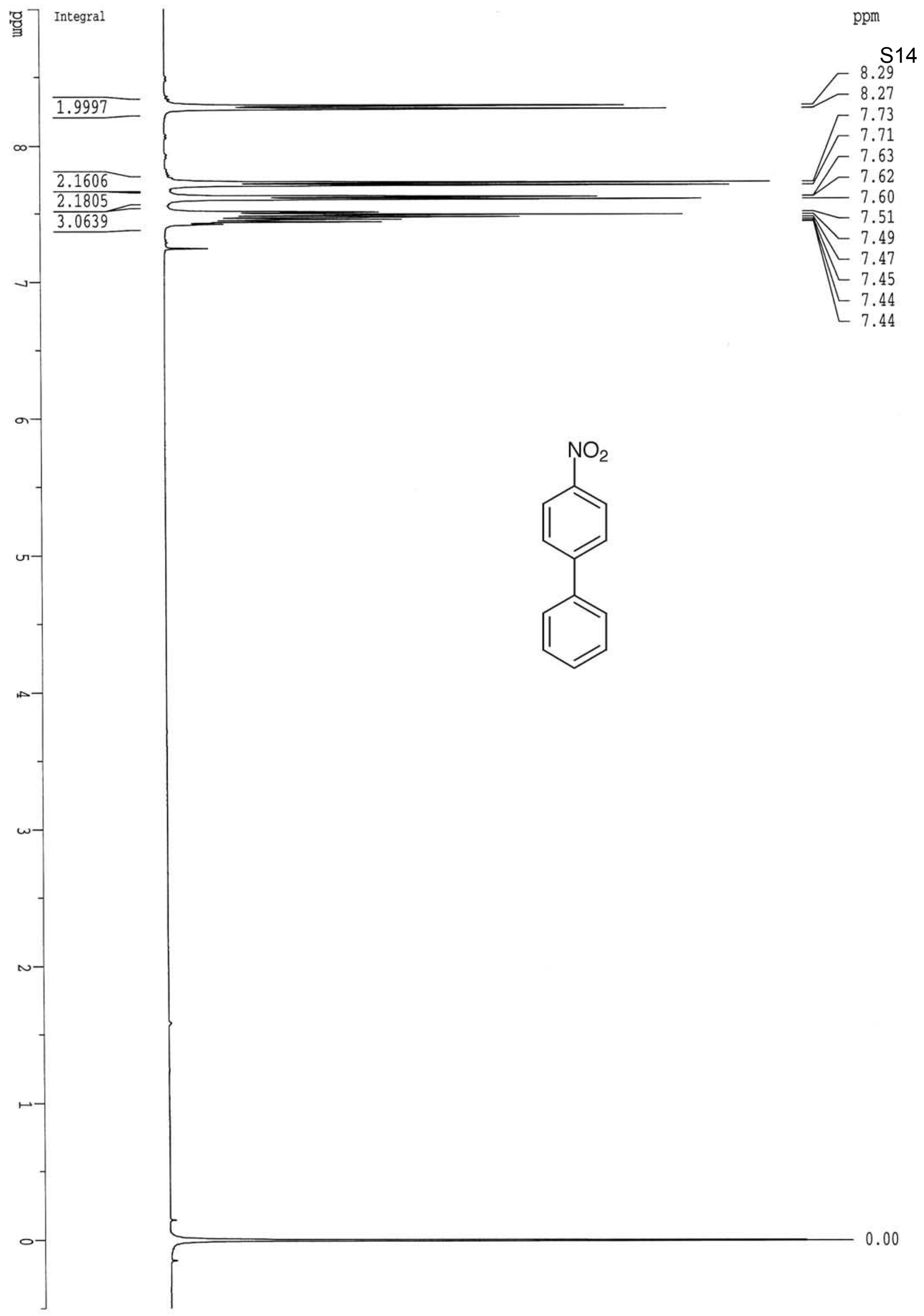



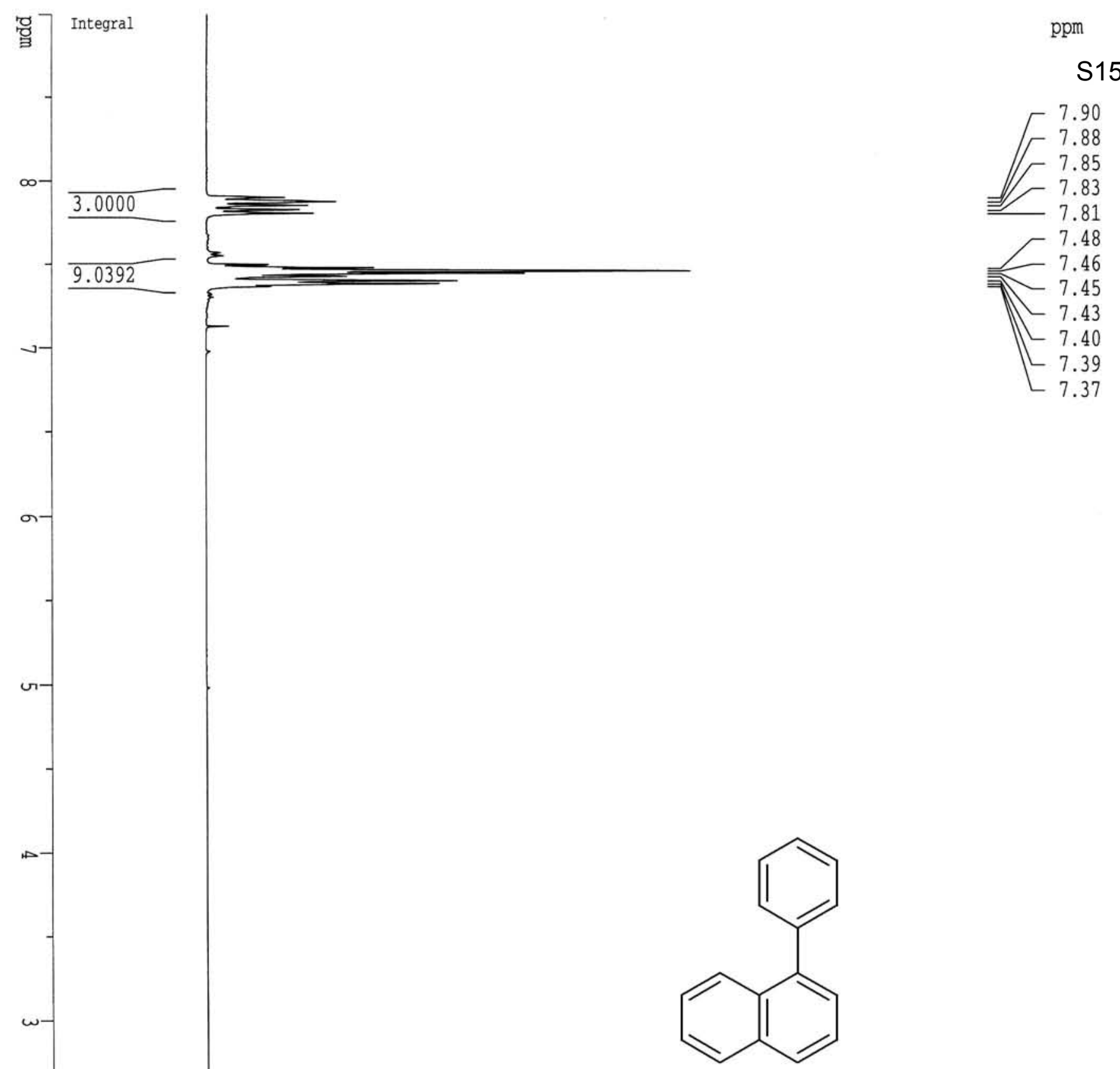


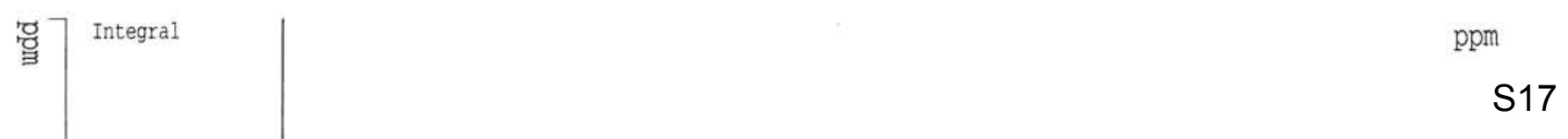

2.0702

$\frac{2.0702}{2.0908}$

1.0442

1.0116

1.0971

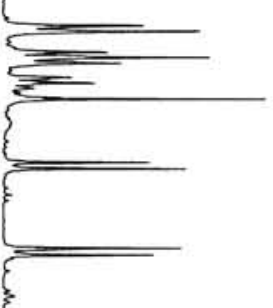

$\begin{array}{r}7.507 \\ -7.490 \\ -7.401 \\ \hline\end{array}$

7.382
-7.359

$-7.259$

7.048

$-7.027$

-6.757
-6.736

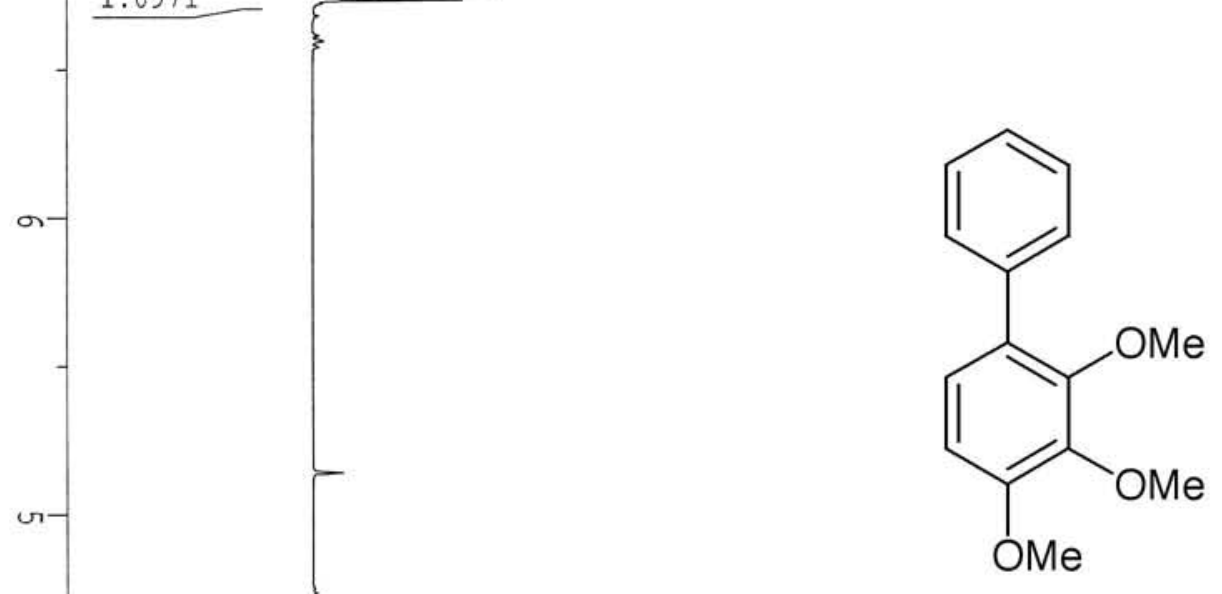

2.9131

3.0656

3.0000

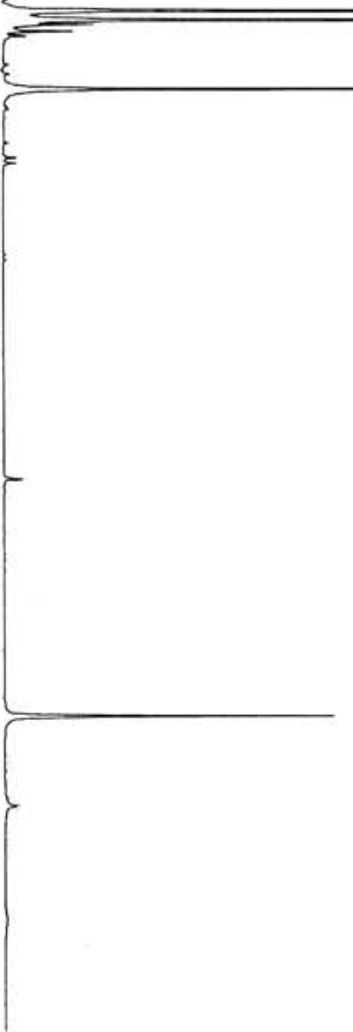

-3.934
-3.904
-3.671 

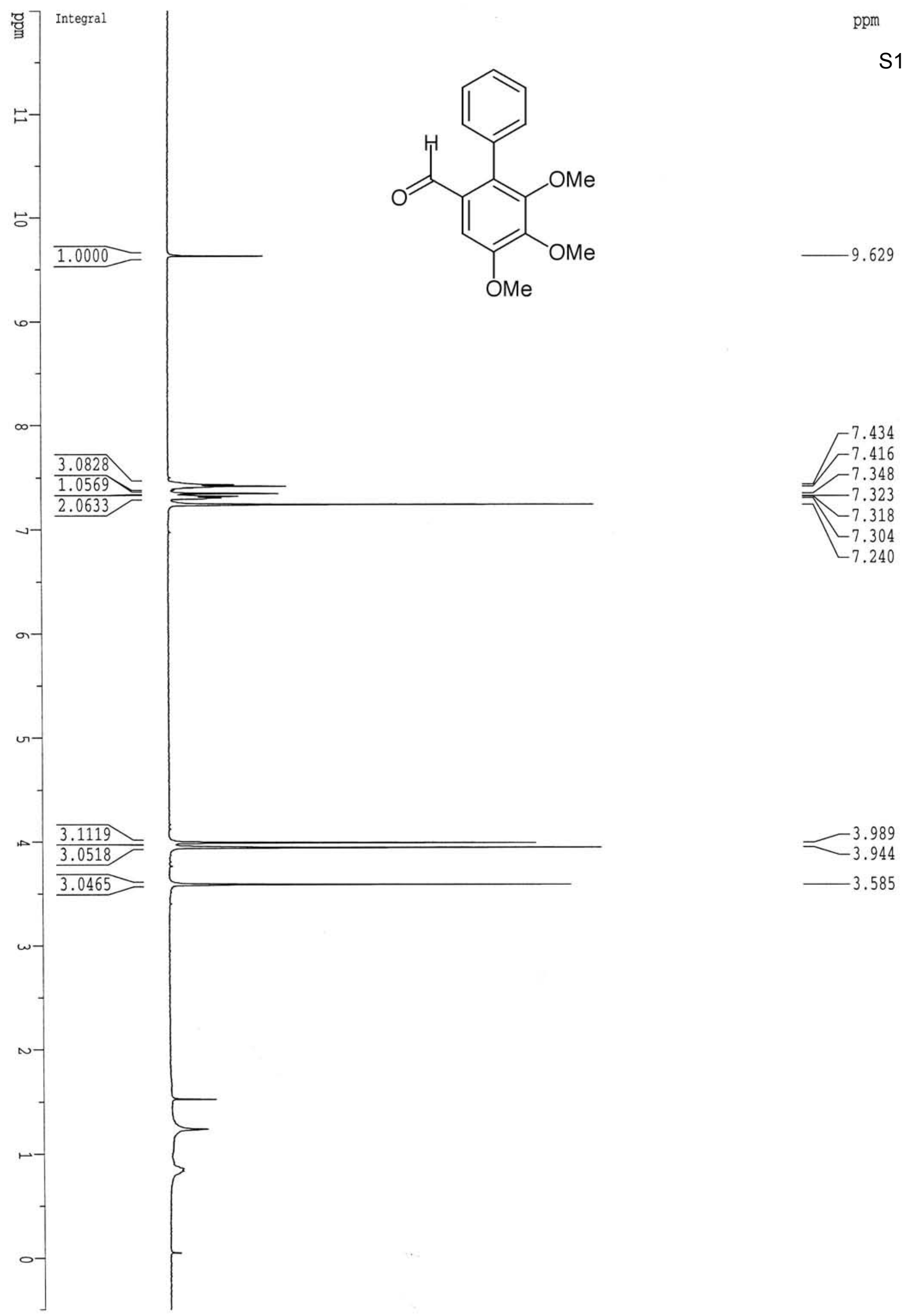

3.989
$-\quad 3.944$ 

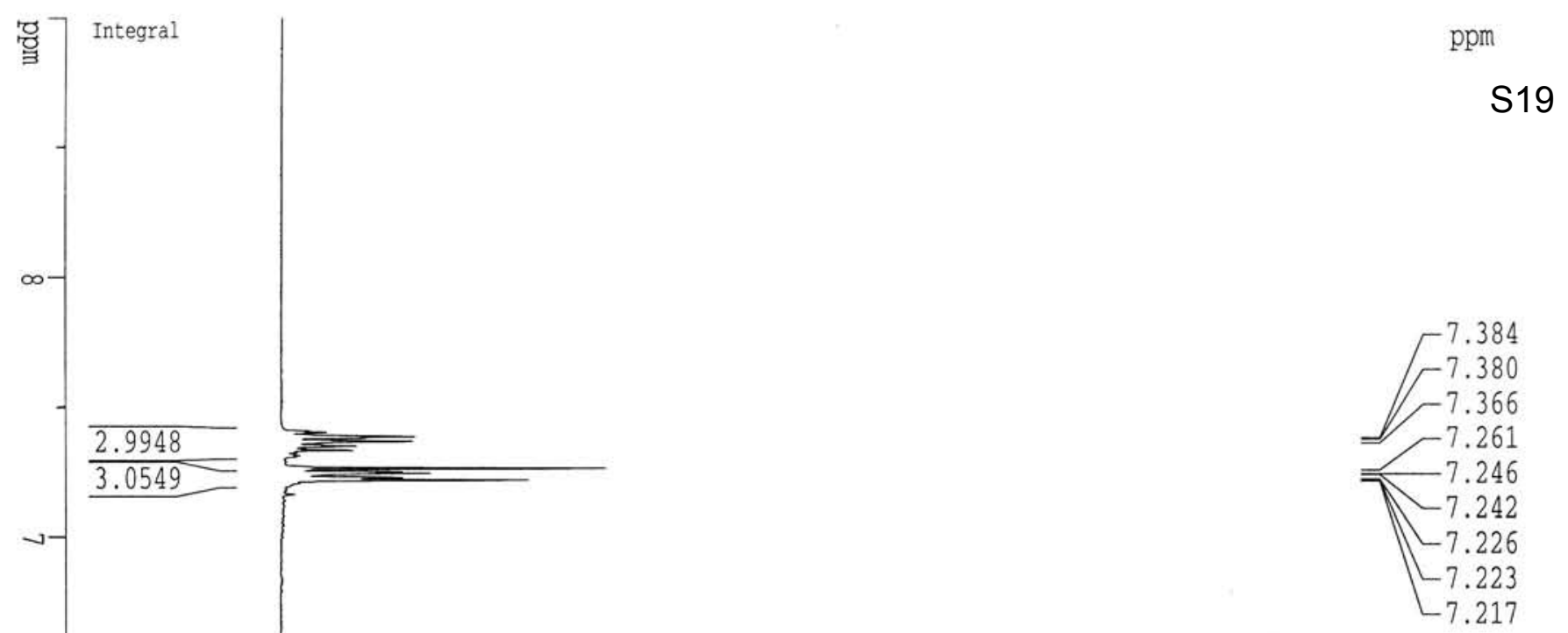<smiles>COC(=O)c1cc(OC)c(OC)c(OC)c1-c1ccccc1</smiles>

\subsection{5} 2.9841

3.0021 $\frac{3.0021}{3.0000}$

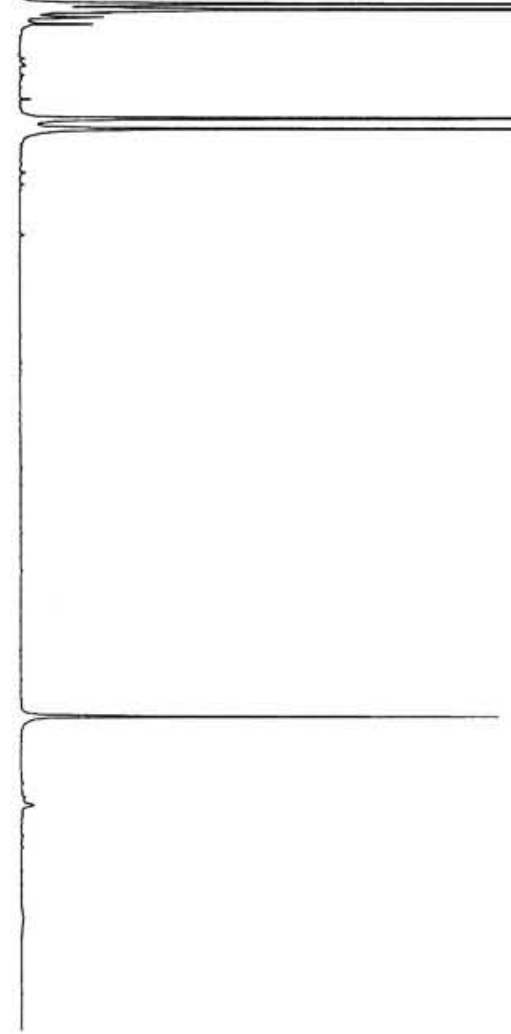

$-3.571$

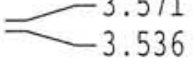




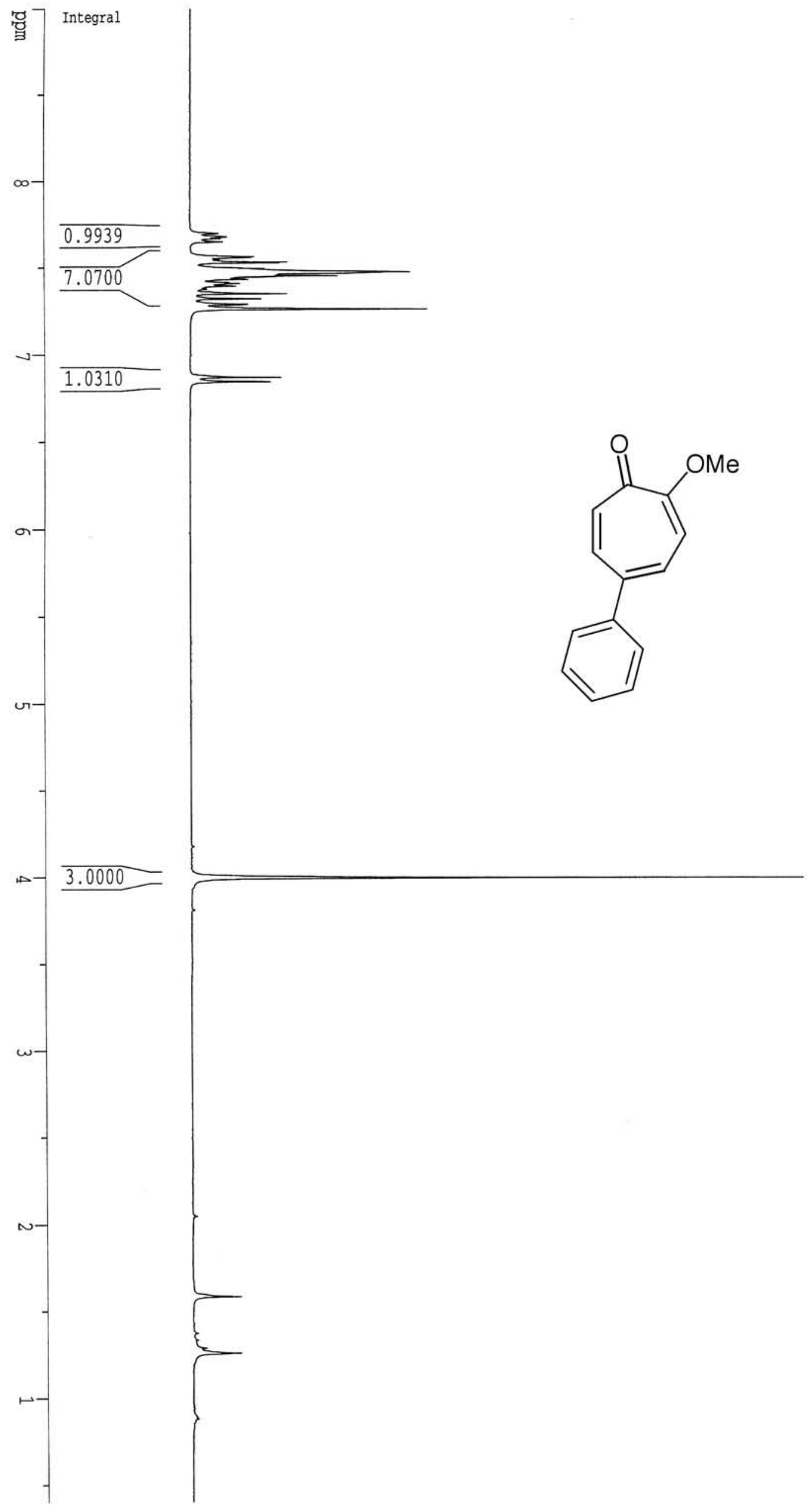

$-7.453$

$-7.434$

$-7.352$

$-7.321$

$-7.291$

-7.286

$-7.262$

$-6.869$

$L_{6.843}$

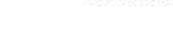




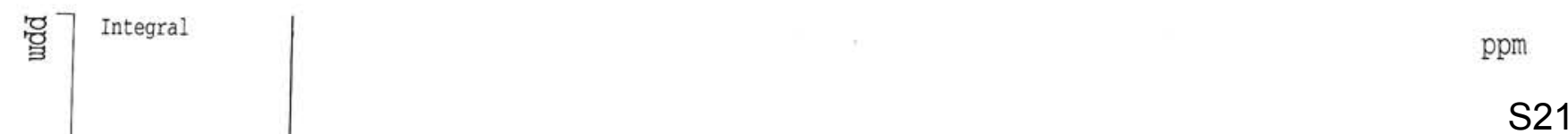<smiles>COc1ccc(Br)c(OC)c1OC</smiles>

\section{$\overline{1.0000}$
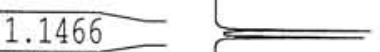

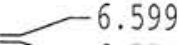

$-7.221$

$-7.198$
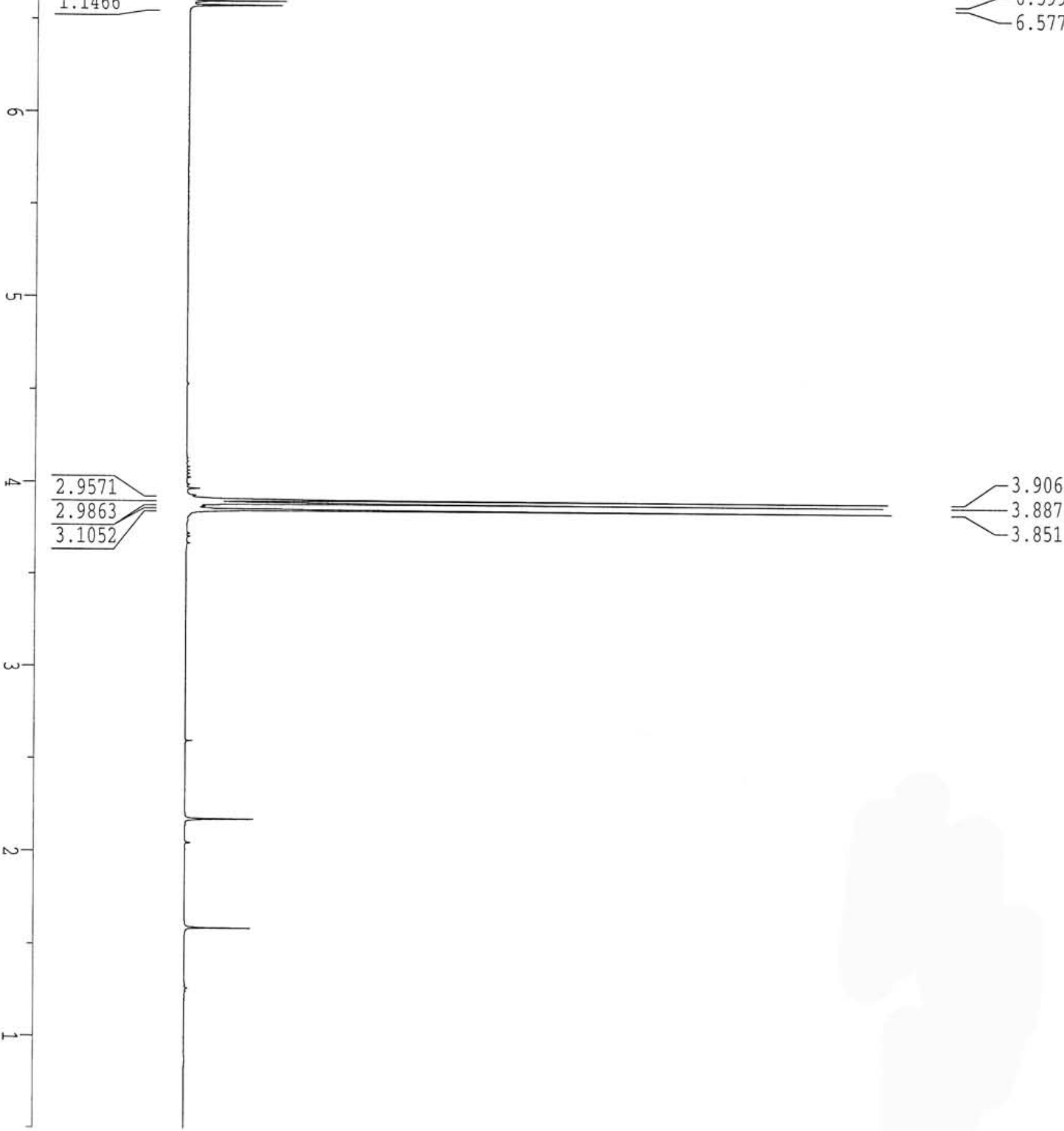

3.906

3.887 